\title{
QUALITY IMPROVEMENT General medicine consultant of the week model shortens hospital length of stay and improves the patient journey
}

\author{
Authors: Amir Aziz, ${ }^{\mathrm{A}}$ April Hawkins, ${ }^{\mathrm{B}}$ Yasmina Gainer, ${ }^{\mathrm{C}}$ Craig Simpson, ${ }^{\mathrm{D}}$ Rachel Barlow ${ }^{\mathrm{E}}$ and Chetan Varma ${ }^{\mathrm{F}}$
}

\section{Background and aims}

The consultant of the week (COW) model of inpatient care means the consultants' primary focus is to deliver ward-based care daily. At Sandwell and West Birmingham Hospitals NHS Trust, a COW model has been successfully used for cardiology and stroke services. This has improved continuity of care and developed a 7-day working week. Our aim was to extend this model to all general medical consultants who manage inpatients.

\section{Methods}

We introduced the COW model to the unselected general medical take. Restructuring of consultant job plans allowed daily ward presence, 5 days per week. Outcome measures included length of stay (LOS) and accuracy of expected date of discharge (EDD).

\section{Results}

LOS over a 12-month period improved from an average of 9.17 days to 6.61 days. The number of EDD changes reduced, from a previous average of 3.0 changes to 1.8 changes. Consultant feedback showed there was an improvement in collaboration between teams, improved training of junior doctors and higher job satisfaction.

\section{Conclusions}

Improved 5-day consultant presence is associated with reduced LOS. Learning points included the delay in implementation due to the complexity of consultant job planning. We plan to extend COW to 7-days for all general medical wards.

KEYWORDS: Service improvement, consultant of the week, length of stay

DOI: 10.7861/fhj.2019-0057

Authors: ${ }^{\text {A }}$ cardiology fellow, Sandwell and West Birmingham NHS Trust, Birmingham, UK; ${ }^{B}$ deputy group director of nursing, Sandwell and West Birmingham NHS Trust, Birmingham, UK; ' head of performance and costing, Sandwell and West Birmingham NHS Trust, Birmingham, UK; ${ }^{D}$ directorate general manager, Sandwell and West Birmingham NHS Trust, Birmingham, UK; ${ }^{E}$ chief operating officer, Sandwell and West Birmingham NHS Trust, Birmingham, UK; ${ }^{\mathrm{F}}$ consultant interventional cardiologist and medicine and emergency group director, Sandwell and West Birmingham NHS Trust, Birmingham, UK

\section{Introduction}

Prolonged lengths of stay in hospital is a major factor in thromboembolic events and hospital-acquired infections. ${ }^{1}$ In addition to this, high bed occupancy rates are associated with increased waiting times of patients in the emergency department (ED) and directly affects patient flow. ${ }^{2}$ Sandwell and West Birmingham Hospitals NHS Trust (SWBH) consists of two teaching hospitals, City Hospital and Sandwell General Hospital, which deliver care to 500,000 people. The average daily take for medicine into the inpatient bed base is between 80-100. This intake leads to daily pressures on the hospital.

The National Institute for Health and Care Excellence (NICE) recommendations are for daily consultant review for all patients admitted with a medical emergency. ${ }^{3}$ Studies looking at daily consultant reviews on patients with acute medical problems found this to be cost-effective with reductions in patient length of stay (LOS). ${ }^{4-10}$

Traditionally at SWBH, a total of 184 beds over eight medical wards (17-25 beds per ward) were supervised by 25 consultants (respiratory, gastroenterology and care of the elderly). Each medical consultant provided two ward rounds (WR) per week on their respective ward (two to three consultants based on each ward). Additional senior WR were provided by specialist registrars with junior doctors performing WR on the other days. This infrequent approach of consultant WR review leads to delayed decisions and discharge. 4,6

We sought out a new way of delivering inpatient care to our medical patients, not only on general medical wards but also in the acute medical unit. The concept of consultant of the week (COW) for general medicine was introduced within our trust. The purpose was to improve the continuity of care provided with improvement in flow, to have patients cared for and treated under the most clinically appropriate consultant teams rather than where there is an available bed, and eventually work towards a 7-day model of care. We report our methodology for implementing these changes and our findings.

\section{Solution/methodology}

A large-scale consultant job planning process via clinical specialty leads was undertaken. This involved reviewing outpatient activity (eg endoscopy and clinics) and how this could be rescheduled to enable consultants to be present on the ward all day, 5 days per 


\begin{tabular}{|c|c|}
\hline 07.00 & $\begin{array}{l}\text { Nurse handover and shift safety } \\
\text { checks completed }\end{array}$ \\
\hline 08.00 & Ward round / board round \\
\hline 09.00 & Discharge 'golden patient' \\
\hline 10.00 & $\begin{array}{l}\text { NIC action check regarding discharges } \\
\text { planned today }\end{array}$ \\
\hline 11.00 & R2G review with COW \\
\hline 12.00 & Feedback from the AMU MDT \\
\hline 13.00 & \\
\hline 14.00 & $\begin{array}{l}\text { Ward huddle to identify next day } \\
\text { discharges }\end{array}$ \\
\hline 15.00 & Confirm next day 'golden patient' \\
\hline 16.00 & $\begin{array}{l}\text { All next day TTAs confirmed and } \\
\text { received / transport confirmed }\end{array}$ \\
\hline 17.00 & \\
\hline 18.00 & All agreed new admissions placed - ward full \\
\hline 19.00 & $\begin{array}{l}\text { Nurse handover and shift safety } \\
\text { checks completed }\end{array}$ \\
\hline 20.00 & \\
\hline 21.00 & \\
\hline 22.00 & $\begin{array}{l}\text { Review all next day discharge documentation } \\
\text { and requirements }\end{array}$ \\
\hline 23.00 & \\
\hline 00.00 & \\
\hline 01.00 & \\
\hline 02.00 & \\
\hline 03.00 & \\
\hline 04.00 & EBMS EDDs updated for the next 48 hours \\
\hline 05.00 & \\
\hline 06.00 & $\begin{array}{l}\text { Today's readiness - patient preparation and } \\
\text { planning }\end{array}$ \\
\hline
\end{tabular}

Fig 1. Medicine wards rhythm of the day. These are the key activities to be undertaken throughout the day. $\mathrm{AMU}=$ acute medical unit; $\mathrm{COW}=$ consultant of the week; EBMS = electronic bed management system; $\mathrm{EDD}=$ expected date of discharge; $\mathrm{MDT}=$ multidisciplinary team meeting; $\mathrm{NIC}=$ nurse-in-charge; $\mathrm{R} 2 \mathrm{G}=$ red to green; TTA $=$ to take away prescriptions

week and free of other clinical commitments. We developed an electronic job-planning spreadsheet to assess supply and demand of all consultant-led activity types. The number of programmed activities were calculated to ensure cover of all activity and, if required, an increase in the number of consultants needed to provide continual acute and elective services.

A COW model of care was introduced which led to daily consultant ward reviews on their base ward and medical outliers. Visual aids were developed to support implementation of the COW model which included COW activity profile and ward-based 'rhythm of the day' (Figs 1 and 2). These provided clarity on the
09.30 Review outliers/referrals

10.30 Ward-based R2G

11.30 AMU MDT

12.30 Ward review and identification of next day discharges and confirmation of today's discharges

14.00

Huddle regarding discharge progress/confirmation same day and next day

$14.00-$

Hot clinic / research / administration / ward attenders

16.00

Next day arrangement/planning confirmation

Fig 2. Consultant of the week activity profile. $A M U$ = acute medical unit; $\mathrm{MDT}=$ multidisciplinary team meeting; $\mathrm{R} 2 \mathrm{G}=$ red to green .

roles of team members. A handover meeting occurred every Friday between COWs to ensure continuity. In order to facilitate consistency, a ward clinical lead model was introduced where the COW could link with other ward leaders (nurse in charge, pharmacy, physiotherapy and social worker) and have a weekly point of contact to discuss process and progress of the COW model. All outlying medical patients were managed by a dedicated outlying team, if this was not possible, then the COW would review. A standard operating procedure was devised for each COW to have a dedicated ward to review.

An important and novel aspect of the COW model is the 11.30am acute medical unit (AMU) multidisciplinary team meeting (MDT) where all consultants attend the board round following the posttake round and accept referrals immediately. This approach aimed to reduce delays in decision making through facilitating specialty review for patients who could be discharged or reviewed following inpatient investigations. Previous studies have not incorporated this in their COW models which makes this change unique.

The main specialties participating in the general medicine COW model were respiratory, gastroenterology and care of the elderly. Specialty COW continued in stroke and cardiology. The COW model was phased in over several months with respiratory starting in November 2017 and gastroenterology completing in January 2018. The change in consultant timetables and the COW was fully implemented on 01 January 2018. The measures chosen to determine outcomes of the COW model were LOS (the patient's entire stay in hospital), increase in morning discharges to support flow (patients discharged before midday) and accuracy of expected discharge dates (EDD; the discharge date is set for the patient to have planned discharged from the acute hospital). The accuracy of the EDD was identified by the number of changes made and the number of patients that were discharged on the date identified. 
As part of the introduction of the COW model, a new dashboard was developed by the trust's informatics and operational teams (supplementary material S1). The dashboard works in real-time and gives the ward up-to-date information on their key performance indicators (KPIs). The introduction of the ward dashboard was part of a wider patient flow improvement programme; in particular, the LOS, number of discharges before midday and patients' EDD. This information was used actively to inform clinical discussions and provided up-to-date information for the COW WRs. One aspect of the 'rhythm of the day' included the 'golden patient'. This was a patient identified for discharge for the following morning at $9 \mathrm{am}$. Discharge letters were completed and transport was confirmed at $4 \mathrm{pm}$ for the following day discharges. This helped improve efficiency and patient flow.

Each specialty clinical lead were asked to comment on the following areas of the implementation of COW: continuity of patient care, follow-up of tests and actions requested for patient care, assessment of EDD and discharge planning, and is there any impact on consultants in terms of job satisfaction.

\section{Outcome}

\section{Quantitative results}

Using a plan, do, study, act (PDSA) approach, it was found for some specialties that a fortnightly COW model was more practical, primarily due to rescheduling of outpatient activity. LOS on all inpatient medical wards over a 12-month period demonstrated a reduction following implementation of the COW model. LOS improved from an average of 9.17 days per patient in 2017 to 6.61 days per patient in 2018. There have been demonstrable improvements with many wards now meeting LOS goals. Thirty-day readmission rates remained stable. Comparison with peer trusts for non-elective LOS (in days) by finished consultant episode were 1.5 days in gastroenterology, 3 days in care of the elderly and 1.1 days in respiratory. We also found reduced average LOS on the AMUs from 29 hours to 24.6 hours for the same period, which may be attributable to the AMU MDT. Variance was seen week-by-week across a 12-month period in pre-midday discharges. The trend of EDD changes appears to be reducing, from a previous average of 3.0 changes per patient in November 2017 to a current average of 1.8 changes per patient. The number of clinics that were cancelled during the winter period across general medicine for each year from 22 in 2017 to eight in 2018 then to zero in 2019.

\section{Qualitative results}

Feedback from each specialty clinical lead were sub-divided into sections:

\section{Patient Experience}

Continuity of care through the COW model could reduce complaints and improve discharge planning. Total number of complaints for medical wards for the period January to May for each year 2017, 2018 and 2019 reduced from 61 to 19 to 13, respectively. Number of complaints due to communication for the same period fell from 21 to 13 to nine, respectively. One consultant said: 'Consistent and regular contact with patients and relatives [allows me to be] able to address simmering issues more promptly.'

\section{Training/education}

There was a positive impact on training and education. By having senior clinicians present daily, this increased and improved wardbased supervision. Junior doctor informal feedback was positive, they felt supported by the COW and were able to easily obtain formal assessments.

\section{Patient flow}

Patient flow improvements were highlighted in the quantitative analysis. In particular, the regular AMU MDT assisted greater understanding of the pressures and challenges experienced across the wider patient care pathway rather than individual departments'/specialties' views. The number of patients being placed in the appropriate specialty bed against age profile improved, with patients over 75 cared for and treated within care of the elderly (where this is deemed to be the most clinically appropriate specialty) rising from $50 \%$ to $80 \%$ which is likely to have a positive impact on patient care and experience. One consultant said: ' [Patient care and experience is improved by] live understanding of what works and doesn't regarding discharge planning.'

Regarding EDD, one consultant commented, 'estimating EDD is a controversial subject in itself, sometimes it is impossible to do [it within the] accuracy of a day in a multimorbid patient with a complicated hospital stay.'

\section{Consultant job satisfaction}

A particular success was the anecdotal improvements around job satisfaction demonstrated through greater communication between consultants, and has improved morale and team working. This was significant because of the implementation was over a busy winter period that challenged all clinical specialties and departments due to high volume, high acuity demand on inpatient beds. Many consultants highlighted that the AMU MDT was an excellent change, with benefits for patient care and greater collaboration with colleagues. Examples of such feedback include:

The reviews and clinical discussion about cases on AMU is fantastic for our patients, they get seen promptly and/or we have a clinical discussion about patients which aids discharge, diagnostics and management. Also, colleagues get a foresight on who is coming to them.

In respiratory, one area of positive feedback from colleagues is the AMU in reach/board round. This allows us to be involved with patients who may need specialist care/tests early on - for example I did a bronchoscopy this afternoon on a patient who probably has tuberculosis on AMU identified yesterday by the respiratory COW, who likely can go home in next 24-28 hours, this may not have happened so quickly pre-COW. There seems an improvement on specialty patients going to specialty ward, though not perfect.

There was a complete change in practice in not needing to cancel elective activities to support the ward, which was prevalent before introduction of COW.

Consultant feedback was mixed regarding the change in job planning, examples include: 'My follow-up clinics have been cut by $25 \%$ but my inflammatory bowel disease follow-up population 
is gradually increasing. This is mirrored across other colleagues' clinics too.'

\section{Discussion}

The purpose for this change was to ensure daily consultant review to improve quality of care and discharge planning for medical patients. A major success of COW was the significant reduction in LOS, with improved continuity of care and a reduction in patient complaints.

There were a number of challenges associated with the revised consultant job plans for the implementation of COW and this required considerable pre-planning. Electronic job planning was undertaken using a demand capacity tool developed inhouse. Implementation of COW was phased and had a PDSA learning cycle. Feedback regarding the challenges of COW that included suggestions for improvement are inherent to the PDSA cycle approach and valuable in evaluation and ongoing model development. Additional medical consultants were recruited (two additional full-time equivalent for both gastroenterology and respiratory medicine) to ensure deliverability of inpatient and outpatient activity. A PDSA approach is being applied to develop the most effective and efficient process to cross cover. As mentioned, consultant feedback was mixed regarding the change in job planning and the cut in follow-up clinics. Follow-up clinics are often not under any targets and therefore may not be prioritised. Other concerns were the COW model leading to increased inpatient activity with no increase in programmed activities in job plans, however this was not the case.

Positive benefits were demonstrated through implementation of COW as shown in the results section. Annualised activity increased subsequent to COW implementation. This is partly likely due to lack of disruption being seen during non-COW weeks. Improvements to training, was an added benefit. Evaluation to date has identified some variability within consultant and ward profiles which requires further investigation to determine reasons for variation and plan for improvement.

Although we did not have data on mortality rates, a previous study showed a significant reduction in mortality rates with daily consultant reviews in gastroenterology with a significantly reduced LOS. ${ }^{4,6}$ However, we are unable to assume that having daily specialist input, especially with the AMU MDT would have an impact on mortality rates, due to multiple variables associated with mortality data and we did not measure this as an outcome.

On reflection, more could have been done in the planning and implementation of the model to improve communication and engaging in wider consultation on the performance matrix. Examples of feedback were: 'Implementation of the components of rhythm of the days needs improving, one consultant managing two wards poses practical problems.' 'The process measures and balance measures were not agreed and therefore the learning is somewhat impaired.' and 'Formal feedback from the stakeholders should be planned for subsequent cycles of change.' This feedback demonstrated that consultants were, in the main, supportive of the COW model with improvements to training an often-cited quality improvement that was not considered in the scope of the change.

\section{Conclusion}

The COW model is normal working practice on general medical wards at SWBH, we have changed from a 'consultant-led' service to a 'consultant-delivered' service. The presence of the COW over 5 days is associated with reduced LOS and improved patient continuity and communication. Continued monitoring of progress against further KPIs will continue to evaluate the positive benefits the COW model can bring and should be considered for implementation in other centres. Plans are in place to extend the COW model to 7 days for all general medical wards.

\section{Supplementary material}

Additional supplementary material may be found in the online version of this article at www.rcpjournals.org/FH]:

S1 - New dashboard showing live analysis including expected date of discharge ward.

\section{References}

1 Clarke A. Why are we trying to reduce length of stay? Evaluation of the costs and benefits of reducing time in hospital must start from the objectivees that govern the change. Qual Heal Care 1996;5:172-9.

2 Cooke MW, Wilson S, Halsall J, Roalfe A. Total time in English accident and emergency departments is related to bed occupancy. Emerg Med J 2004;21:575-6.

3 National Institute for Health and Care Excellence. Chapter 26 Frequency of consultant review. In: Emergency and acute medical care in over 16s: service delivery and organisation: NICE guideline [NG94]. NICE, 2018.

4 Ahmad A, Purewal TS, Sharma D, Weston PJ. The impact of twicedaily consultant ward rounds on the length of stay in two general medical wards. Clin Med 2011;11:524-8.

5 Bray BD, Ayis S, Campbell J et al. Associations between stroke mortality and weekend working by stroke specialist physicians and registered nurses: prospective multicentre cohort study. PLoS Med 2014:11:e1001705.

6 Singh S, Lipscomb G, Padmakumar K et al. Daily consultant gastroenterologist ward rounds: reduced length of stay and improved inpatient mortality. Frontline Gastroenterol 2012;3:29-33.

7 Fisher AA, Davis MW, Rubenach SE et al. Outcomes for Older Patients With Hip Fractures: The Impact of Orthopedic and Geriatric Medicine Cocare. J Orthop Trauma 2006;20:172-8.

8 Kerlin MP, Small DS, Cooney E et al. A Randomized Trial of Nighttime Physician Staffing in an Intensive Care Unit. N Engl ] Med 2013;368:2201-9.

9 Rich EC, Gifford G, Luxenberg M, Dowd B. The relationship of house staff experience to the cost and quality of inpatient care. JAMA 1990;263:953-7.

10 Pethybridge J. How team working influences discharge planning from hospital: a study of four multi-disciplinary teams in an acute hospital in England. J Interprof Care 2004;18:29-41.

Address for correspondence: Dr Chetan Varma, Birmingham City Hospital, Sandwell and West Birmingham NHS Trust, Dudley Road, Birmingham B18 7QH, UK.

Email: c.varma@nhs.net 